\title{
Gynaecological Examination and Equipment in the Surgery
}

\author{
J. A. HENDERSON
}

British Medical fournal, 1971, 1, 273-274

Obviously the choice of equipment for a doctor's surgery is a highly individual matter, based on experience, taste, and prejudice. What follows is a personal choice based on fifteen years' experience in general practice, in which a good deal of gynaecology and obstetrics has been involved.

It goes without saying that a good examination couch, a sterilizer, and adequate lighting are almost certainly part of the general equipment of any surgery. The days of the gynaecological couch with stirrups have, quite rightly, passed away; it was both uncomfortable and undignified and left the shy, embarrassed patient quivering with mortification. A perfectly adequate examination can be carried out by the patient lying on her back with heels together and knees apart, or, for better exposure of the fourchette and perineum, with her knees pulled well up on to her chest and held apart in that position, or lying in the left lateral position. It is worth having a good light immediately above the couch and preferably, but not essentially, a light on a movable stand that can be wheeled up as an auxiliary lighting source. Some sort of table to accommodate equipment is also needed (one fitted with glass shelves and big castors is the most luxurious).

\section{Examination Equipment}

Disposable plastic gloves are an indisputable blessing and have replaced the messy, miserable job of washing, drying, and possibly sterilizing rubber ones. The former can be obtained in varying sizes, either sterile or unsterile. For most

\section{London S.W.3}

J. A. HENDERSON, M.R.c.s., M.R.c.o.G., General Practitioner gynaecological examinations unsterile gloves seem to me perfectly adequate, but with an open cervix (during a miscarriage or immediate post partum) or for any intrauterine procedure sterile gloves and a full sterile technique should be used.

A lubricant cream is needed for both manual examination and for passing a speculum. I used to use Dettol obstetric cream until several patients complained of soreness after examination, since when I have changed to K.Y. jelly, which has the added advantage that if a culture of the vaginal flora is desirable it contains no bactericidal chemicals.

SPECULA

The next logical step in any gynaecological examination after inspection of the abdomen and palpation both of the abdomen and vagina, including bimanual examination, is inspection of the vagina and cervix. There are many specula available, and I can only express my own personal preference.

Brewer's bi-valve speculum fulfils most of the requirements for surgery examinations. The simplest method of illumination is to use a plain metal speculum and have a separate light source. As a rule this is adequate for inspection, but the light may be hard to manipulate if vaginal or intrauterine manipulation is envisaged. Probably the most practical is a Coldlite speculum (Brewer's pattern) which has an illuminated lower blade; this gives a good light and good exposure. A transformer is necessary for this, but it will give an outlet for a cautery in addition to the light supply for the speculum, and the cautery itself forms a valuable addition to the equipment of any surgery. Perhaps the ultimate in speculum illumination is provided by a Fibrelight cable illuminated from an independent remote source.

I have a small Sims duck-billed speculum too, which can be very useful for both ends of the age scale-the young girl 
with the intact hymen, or the elderly lady with the narrowed atrophic vagina. It does not give nearly as good an exposure as the Brewer type, but you can take a swab and catch a glimpse without too much discomfort or trauma.

\section{OTHER GENERAL EQUIPMENT}

Several kidney dishes, small enough to fit readily into the sterilizer but big enough to accommodate the larger instruments without undue overlap, are necessary. I find I can perform quite reasonable sterile procedures by taking instruments direct from sterilized kidney dishes without the need for sterile towels on which to lay them, though supplies of sterilized disposable paper towels are extremely useful.

One or two pairs of sponge holders are needed for swabbing out the vagina as well as supplies of sterile gauze swabs. A solution of cetrimide seems to me as good a sterilizing agent as any for the vulva and vagina before any intrauterine manoeuvre.

A pair of vulsellum forceps (Schroeder's single toothed) can usually be inserted into the cervix to steady it without pain and can be invaluable for passing small dilators (Hegar's double ended $\frac{1}{2}$ and $\frac{3}{4}$ ) which may be needed before the introduction of an intrauterine device for contraceptive purposes.

For obtaining pathological material swabs and slides are obviously necessary in addition to the Ayre spatulae for Papanicalaou smears and the fixative for the slides and small slide boxes for transport to the laboratory. I use slides with ground glass ends on which the patient's name can be written and, as a rule, take two slides in each case. The fixative consists of a wax solution and can be supplied in a plastic drop bottle which is convenient and economical.

\section{SWABS}

As far as swabs of vaginal discharges are concerned I am sure it is best to examine these directly with a microscope in the surgery, and both trichomonal and candidial infections can be identified there and then. With the extremely efficient and handy laboratory facilities in the centre of London, however, I tend to ask for a professional report. I usually send a dry swab and one in a solution of trichomonas medium, together with a slide prepared on the spot. If the patient takes those herself direct to the laboratory it ensures that a fresh preparation can be examined; I can thus obtain a report of direct microscopy within a few hours and of culture and sensitivities twenty-four hours later.

\section{Specialized Equipment}

\section{CERVICAL EROSIONS}

Treatment of cervical erosions can often be easily tackled as what our American friends would call an "office procedure." I use silver nitrate points held in a special holder; it is worth remembering that though the cervix may be reasonably insensitive the upper vagina certainly is not, and any excessive moisture that falls on the vaginal vault will be quite uncomfortable, so it is kind to dab the treated cervix with a gauze swab on a sponge holder before the speculum is taken out and the patient sent away. The electric cautery can also be used with caution in short spells, but if allowed to remain red hot at or near the cervix for any length of time it can be painful. Perhaps it is superfluous to add that a Papanicalaou smear should, in my view, be always taken before the treatment of any erosion is started.

\section{CONTRACEPTION}

Most general practitioner gynaecologists should be capable of giving modern contraceptive advice. As far as intrauterine devices are concerned I find that the SAF-T-CoIL is generally very satisfactory and in parous women can usually be fitted without anaesthesia but, of course, with a full aseptic technique. As an added precaution for those patients whom I fit with a device in my own surgery I prescribe a subsequent four-day course of tetracycline.

In all cases where difficulty or pain is encountered in passing small Hegar dilators, or in cases where for one reason or another a Gräffenberg ring appears desirable (small uterus, nulliparity, or previous difficulties with a coil), general anaesthesia should be employed. This should best be carried out in a proper operating theatre with all the added equipment needed for a safe anaesthetic.

A set of fitting rings for contraceptive pessaries together with a supply of varying sizes of caps and introducers are part of my equipment, and I also have some sets of glass vaginal dilators. Recently, the almost universal use of vaginal tampons and the advent of the permissive society seem to have reduced the need for using vaginal dilators. Certainly until 10 or 15 years ago watchspring pessaries and even Hodge's pessaries in innumerable sizes used to litter the shelves of my cupboard. Now I seem to have so few patients with prolapse needing treatment with pessaries I just keep their own particular size of watchspring pessary in stock, and I have not inserted a Hodge's pessary for as long as I can remember.

\section{PREGNANCY TESTING}

It is now so simple to carry out pregnancy testing in the surgery that equipment for this has become almost as routine as the more traditional colorimetric urine test strips. A latex type of pregnancy test apparatus is an extremely useful addition to surgery equipment, and a diagnosis can be given in two or three minutes, whereas if the urine specimen were sent to a laboratory it would be both costly in time and money.

\section{Chaperones}

Finally, there is the question of chaperones. Though obviously the situation must vary from practice to practice and doctor to doctor, I have never employed one. This is not to say that there have not been secretaries in neighbouring rooms, but I have never found the need to have another woman in the room when I have examined a female patient. The presence of a third party may deny the doctor some vital confidence, which from time to time may be of enormous help not only in reaching a diagnosis but in unburdening a worried mind. Possibly I am lucky in having a practice where medicine comes first and modesty and prudishness comes a bad second, or that $I$ see women in a higher intelligence group than the average. Nevertheless, I believe that the whole doctor-patient relationship is based on trust and whether your practice involves the intimacy of gynaecological examination, or whether it does not, a score of chaperones would not make up for loss or lack of trust, and if trust is there no chaperone is needed. 